\title{
ARTICLE
}

Acute myeloid leukemia

\section{Sequential high-dose cytarabine and mitoxantrone (S-HAM) versus standard double induction in acute myeloid leukemia-a phase 3 study}

\author{
Jan Braess ${ }^{1,2} \cdot$ Susanne Amler ${ }^{3,4} \cdot K$ Karl-Anton Kreuzer ${ }^{5} \cdot K_{\text {Karsten Spiekermann }}^{2} \cdot$ Hans Walter Lindemann $^{6}$. \\ Eva Lengfelder ${ }^{7} \cdot$ Ullrich Graeven $^{8} \cdot$ Peter Staib $^{9} \cdot$ Wolf-Dieter Ludwig $^{10} \cdot$ Harald Biersack $^{11} \cdot$ Yon-Dschun Ko $^{12}$. \\ Michael J. Uppenkamp ${ }^{13} \cdot$ Maike De Wit $^{14} \cdot$ Stefan Korsten $^{15} \cdot$ Rudolf Peceny $^{16} \cdot$ Tobias Gaska $^{17} \cdot$ Xaver Schiel $^{18}$. \\ Dirk M. Behringer ${ }^{19} \cdot$ Michael G. Kiehl $^{20}$ - Bettina Zinngrebe ${ }^{21} \cdot$ Gerald Meckenstock $^{22} \cdot$ Eva Roemer $^{23}$. \\ Dirk Medgenberg $^{24} \cdot$ Ernst Spaeth-Schwalbe ${ }^{25} \cdot$ Gero Massenkeil $^{26} \cdot$ Heidrun Hindahl $^{27} \cdot$ Rainer Schwerdtfeger $^{28}$. \\ Guido Trenn ${ }^{29}$ - Cristina Sauerland ${ }^{3} \cdot$ Raphael Koch $^{3} \cdot$ Martin Lablans $^{3,30} \cdot$ Andreas Faldum $^{3} \cdot$ Dennis Görlich $^{3}$. \\ Stefan K. Bohlander ${ }^{2,31}$ - Stephanie Schneider ${ }^{2}$ - Annika Dufour ${ }^{2}$ - Christian Buske ${ }^{2,32} \cdot$ Michael Fiegl $^{2}$. \\ Marion Subklewe ${ }^{2} \cdot$ Birgit Braess $^{1,2} \cdot$ Michael Unterhalt $^{2} \cdot$ Anja Baumgartner $^{2} \cdot$ Bernhard Wörmann $^{33}$. \\ Dietrich Beelen ${ }^{34} \cdot$ Wolfgang Hiddemann ${ }^{2} \cdot$ for the AML-CG
}

Received: 16 May 2018 / Revised: 26 July 2018 / Accepted: 9 August 2018 / Published online: 1 October 2018

(c) The Author(s) 2018. This article is published with open access

\begin{abstract}
Dose-dense induction with the S-HAM regimen was compared to standard double induction therapy in adult patients with newly diagnosed acute myeloid leukemia. Patients were centrally randomized (1:1) between S-HAM (2nd chemotherapy cycle starting on day $8=$ "dose-dense") and double induction with TAD-HAM or HAM(-HAM) (2nd cycle starting on day 21 = "standard"). 387 evaluable patients were randomly assigned to S-HAM $(N=203)$ and to standard double induction $(N$ $=184)$. The primary endpoint overall response rate (ORR) consisting of complete remission $(\mathrm{CR})$ and incomplete remission $\left(\mathrm{CR}_{\mathrm{i}}\right)$ was not significantly different $(P=0.202)$ between S-HAM $(77 \%)$ and double induction $(72 \%)$. The median overall survival was 35 months after S-HAM and 25 months after double induction $(P=0.323)$. Duration of critical leukopenia was significantly reduced after S-HAM (median 29 days) versus double induction (median 44 days) $-P<0.001$. This translated into a significantly shortened duration of hospitalization after S-HAM (median 37 days) as compared to standard induction (median 49 days) $-P<0.001$. In conclusion, dose-dense induction therapy with the S-HAM regimen shows favorable trends but no significant differences in ORR and OS compared to standard double induction. S-HAM significantly shortens critical leukopenia and the duration of hospitalization by 2 weeks.
\end{abstract}

\section{Introduction}

Even though treatment of acute myeloid leukemia (AML) with curative intent is not standardized worldwide, it always

In memory of Thomas Büchner (born 1934 in Berlin—died 2016 in Münster)—founder of the German Acute Myeloid Leukemia Cooperative Group (AML-CG).

Electronic supplementary material The online version of this article (https://doi.org/10.1038/s41375-018-0268-9) contains supplementary material, which is available to authorized users.

Jan Braess

Jan.Braess@barmherzige-regensburg.de

Extended author information available on the last page of the article involves intensive induction chemotherapy with the aim of reaching a (morphologically) complete remission (CR) and subsequent risk stratified post-remission therapy. Except for acute promyelocytic leukemia (APL) [1] and-recentlyFLT3 mutated AML [2], the substantial (genetic) heterogeneity of AML [3, 4] so far has had only a moderate impact on the choice of therapy. Specifically, the increased knowledge of AML biology has not yet obviated the necessity for intensive cytotoxic chemotherapy. This is especially true for the initial induction phase where leukemia burden reduction by 3-4 logs and achievement of a complete (morphological) remission-following a prolonged period of deep aplasia-is the main goal.

One current standard induction therapy approach-as established by the AML-CG and many other cooperative 
groups in the last decades-is "double induction" (DI) therapy [5-8]. This strategy consists of two cycles of intensive cytarabine (AraC) and anthracycline/anthracenedione-based chemotherapy. In younger patients ( $<60$ years), the second chemotherapy cycle is given mandatorily on day 21 even if no residual blasts are detected in a bone marrow aspirate on day 16 ("mandatory DI"). In older patients ( $\geq 60$ years), the second cycle is only given in case of residual blasts at this time point ("conditional DI"). In an attempt to further improve this approach, a time-sequential modification of the high-dose AraC/mitoxantrone combination ( $\mathrm{S}$ HAM) was developed into a dose-dense application of highdose AraC (HD-AraC) on days 1 and 2 followed by mitoxantrone on days 3 and 4 and a repetition of the same sequence after a 3-day treatment-free period from days 8 to 11. Hence, the S-HAM regimen covers a total of 11 days of treatment only. With this regimen, highly encouraging results were obtained for salvage therapy in relapsed and refractory AML [9]. Hence, the AML-CG explored this dose-dense concept subsequently in first-line therapy [10]. The AML-CG 2004 pilot trial demonstrated the feasibility and high efficacy of the S-HAM regimen without increasing toxicity and revealed a substantial reduction in the duration of critical leukopenia. The current AML-CG 2008 study was initiated to confirm these results and to compare the efficacy and toxicity of S-HAM with standard double induction within a prospective randomized trial using the overall response rate (ORR) as the primary endpoint.

Secondary endpoints were overall survival (OS), the duration of critical cytopenias, and the duration of hospitalization, amongst others.

\section{Patients and methods}

\section{Study conduct}

The current study was carried out in accordance with the declaration of Helsinki. All patients gave their consent after having been informed about the purpose and the investigational nature of the trial. Before initiation, the study received approval of the responsible institutional review board and the ethics committees of the participating institutions. The clinical study is an official study of the "Kompetenznetz Akute und Chronische Leukämien" and is registered in the European Trial Registry as EudraCT 2007-003103-12.

\section{Patients}

Patients aged $\geq 18$ years with newly diagnosed AML including de-novo AML and secondary AML after a preceding hematological disorder could be included. Patients with APL were excluded. There was no upper age limit, but patients needed to be considered fit for intensive AML therapy by their treating physician.

\section{Induction therapy}

For induction therapy, eligible patients were randomly assigned to standard double induction or S-HAM with stratifications for de novo or secondary AML and age $<60$ or $\geq 60$ years. Within the group of patients between 60 and 70 years of age, a distinction was made according to biologic rather than chronologic age, i.e., patients of ages 60-70 years who were considered biologically "young" were included into the "young" population while biologically "old" patients were included into the "old" treatment group. This discrimination was applied for the selection of standard induction which was TAD-HAM in younger patients and HAM-(HAM) in older patients, and was compared to SHAM as the experimental arm. The allocation to the "young" or "old" group was done at the discretion of the treating physician and was performed at registration/randomization. Please note that only chronological age was used for dose determination in HD-AraC components-i.e., $3 \mathrm{~g} /$ $\mathrm{m}^{2}$ for patients $<60$ years and $1 \mathrm{~g} / \mathrm{m}^{2}$ for patients $60+$ years.

\section{Standard double induction in younger patients}

Standard double induction in younger patients consisted of TAD 9 followed by HAM on day 21. The TAD-9 regimen comprised a continuous infusion of AraC at $100 \mathrm{mg} / \mathrm{m}^{2}$ per day for the first $48 \mathrm{~h}$ followed by short infusions of $\operatorname{AraC}\left(100 \mathrm{mg} / \mathrm{m}^{2}\right)$ twice daily on days $3-8$. Daunorubicin was applied on days $3-5$ at $60 \mathrm{mg} / \mathrm{m}^{2}$ as a $1 \mathrm{~h}$ infusion and thioguanine was given orally $\left(100 \mathrm{mg} / \mathrm{m}^{2}\right)$ twice daily on days 3-9. Seven days after the completion of the first cycle (i.e., on day 16 after the start of TAD-9), a bone marrow aspirate was taken to evaluate the degree of leukemic cytoreduction. The second cycle of double induction was started on day 21 irrespective of peripheral blood counts and irrespective of the blast count in the bone marrow aspirate on day 16 ("mandatory" DI). For the second cycle, the HAM regimen was applied consisting of HD-AraC at 3 $\mathrm{g} / \mathrm{m}^{2}\left(1 \mathrm{~g} / \mathrm{m}^{2}\right.$ for patients $60+$ years chronological age $)$ as a $3 \mathrm{~h}$ infusion every $12 \mathrm{~h}$ on days $1-3$. Mitoxantrone was applied at a dose of $10 \mathrm{mg} / \mathrm{m}^{2}$ as $1 \mathrm{~h}$ infusion on days $3-5$.

\section{Standard induction in older patients}

Standard induction in older patients consisted of HAM followed by the second HAM cycle on day 21 only in case of an inadequate leukemic cytoreduction ( $>5 \%$ residual blasts in the bone marrow smears) after the first HAM cycle ("conditional" DI). 
Treatment regimen for Younger Patients $[<60$ (- 70) Years]

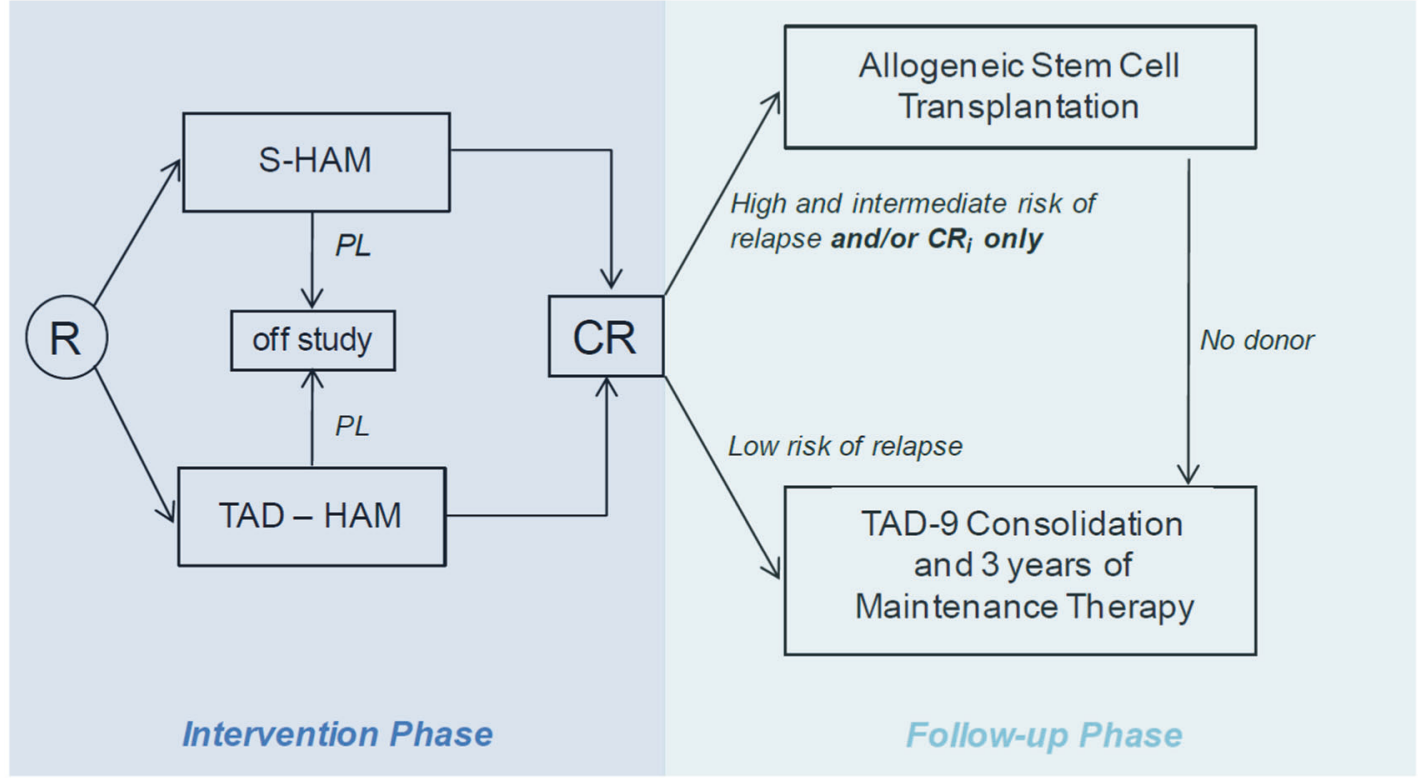

Flow chart for younger patients $[<60$ (- 70) years] if no contraindications against allogeneic transplantation in CR-1; R: randomisation, PL: persistent leukemia, CR: complete remission

\section{Treatment regimen for Elderly Patients [ $\geq 60$ (- 70) Years]}

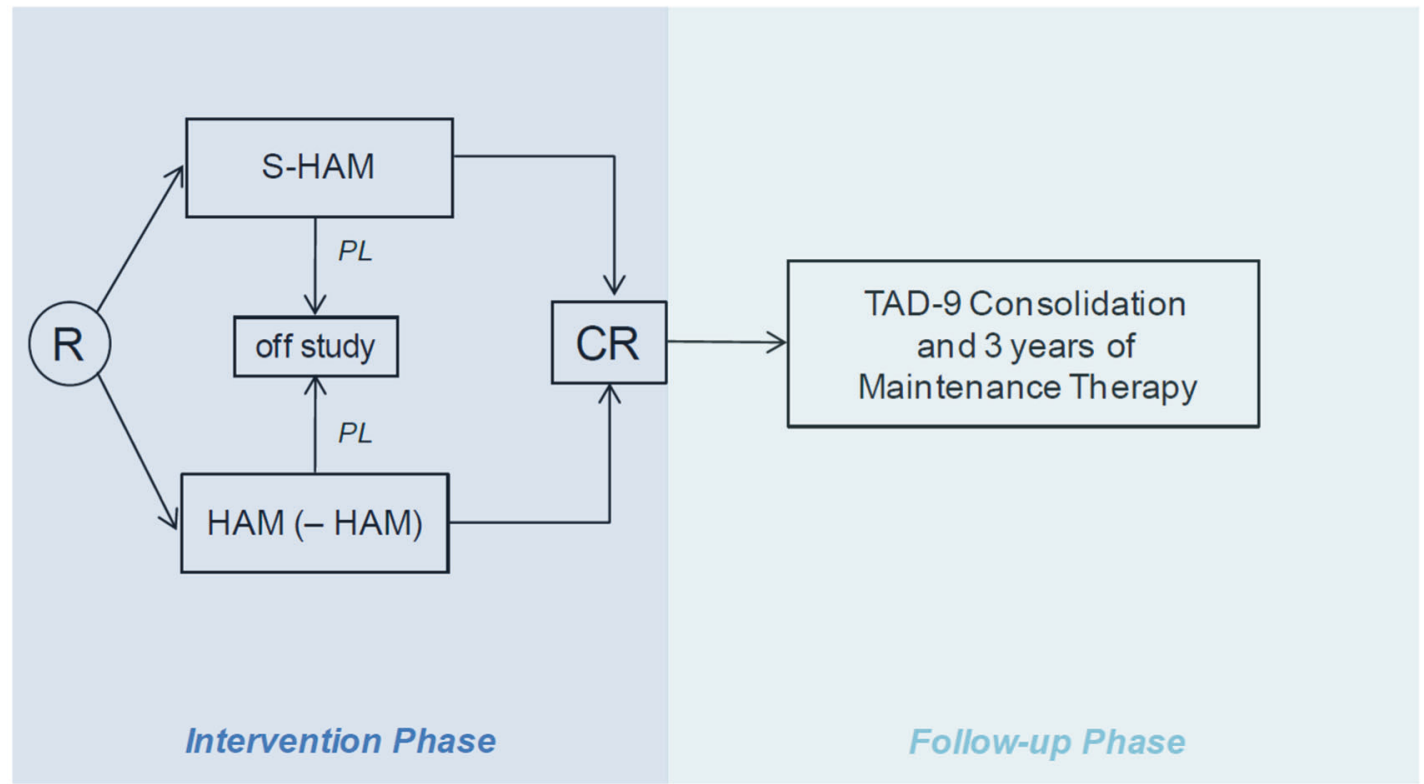

Flow chart for elderly patients $[\geq 60(-70)$ years], who are not potentially eligible for allogeneic transplantation in CR-1; R: randomisation, PL: persisten leukemia, CR: complete remission

Fig. 1 Flow chart of protocol treatment

\section{The S-HAM regimen}

The S-HAM regimen consisted of HD-AraC at a dose of $3 \mathrm{~g} / \mathrm{m}^{2}\left(1 \mathrm{~g} / \mathrm{m}^{2}\right.$ for patients age $60+$ years chronological age) as a $3 \mathrm{~h}$ infusion every $12 \mathrm{~h}$ on days $1-2$ and days 8 9. Mitoxantrone was applied at a dose of $10 \mathrm{mg} / \mathrm{m}^{2}$ as a 1 $\mathrm{h}$ infusion on days 3-4 and 10-11. Two to seven days after completion of the S-HAM regimen (i.e., on days 
Fig. 2 Study flow diagram (CONSORT)

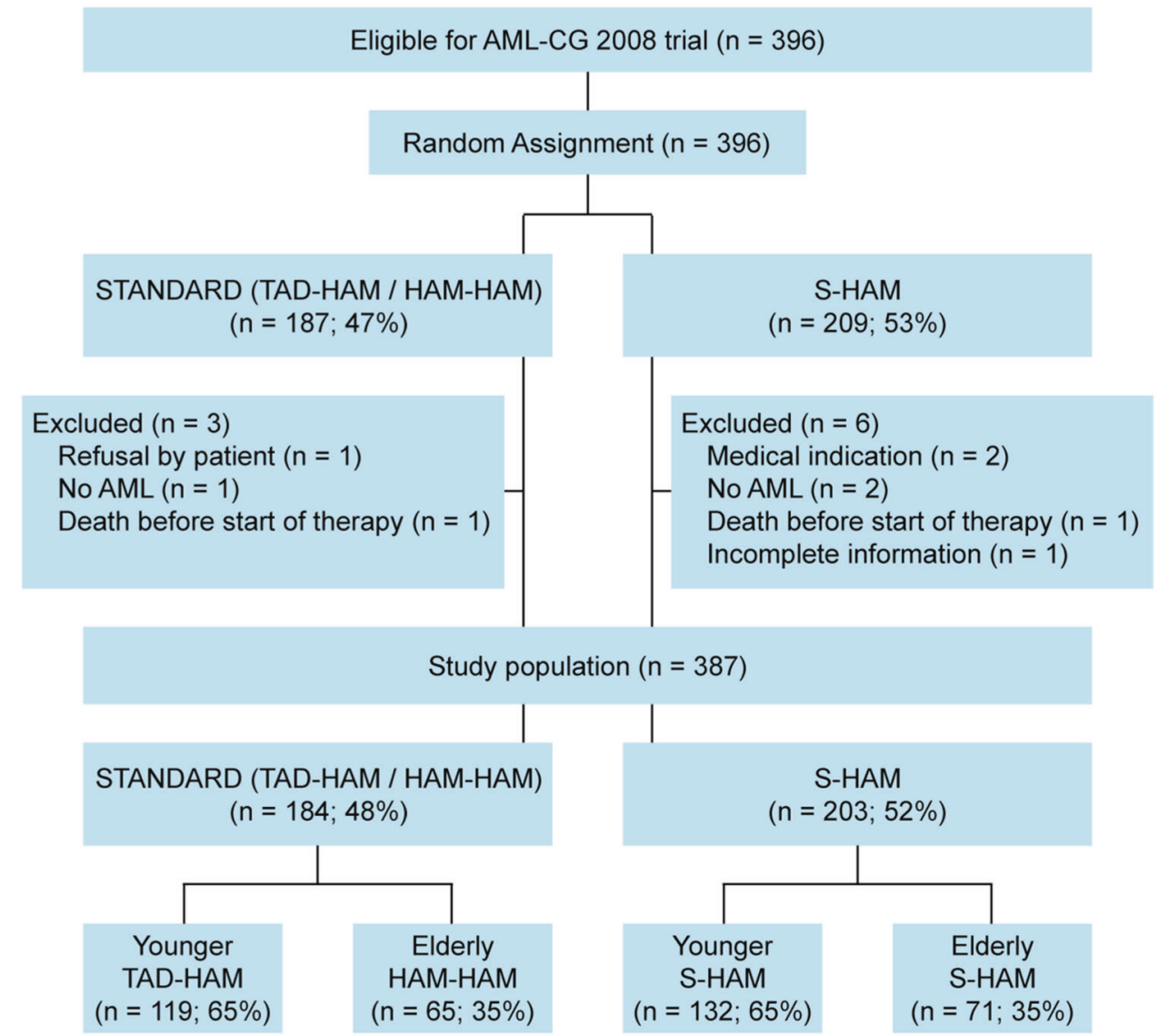

13-18 after the start of S-HAM), a bone marrow aspirate was obtained to determine the degree of leukemic cytoreduction.

The flow chart of the study is depicted in Fig. 1.

\section{Supportive care during induction}

Standard supportive measures were applied including antifungal prophylaxis with posaconazole. Growth factor support after the completion of induction therapy was mandatory after S-HAM with pegylated G-CSF (6 mg s.c.) on day 18 if there were no residual blasts in the posttreatment bone marrow aspirate. After standard double induction, G-CSF application was optional if no residual blast had been observed in the day 16 bone marrow aspirate. This was also possible in "older" patients receiving the second cycle due to prior insufficient blast clearance after the first cycle.

\section{Postremission therapy}

Patients in CR after induction therapy were placed on consolidation treatment with one cycle of TAD and 3 years of monthly myelosuppressive maintenance with alternating 5-day cycles of AraC plus thioguanine, daunorubicin, or cyclophosphamide (AD-AT-AD-AC...) [11, 12] in patients considered to be at a low risk of relapse or not eligible for allogeneic transplantation. Eligible patients with a high or intermediate risk of relapse were allocated to allogeneic transplantation in first remission (CR-1).

The formal interventional phase of the present study involved only the induction period until the response to treatment could be evaluated with a maximum of 90 days after the start of induction treatment. Postremission treatment was defined but was not part of the formal study.

\section{Endpoints and definitions}

The primary endpoint of the study was the ORR comprizing $\mathrm{CR}$ and $\mathrm{CR}_{\mathrm{i}}$ (complete remission with incomplete peripheral recovery) [13] after the completion of induction treatment. CR was documented after normalization of peripheral blood counts by a bone marrow aspirate immediately prior to consolidation therapy. Secondary endpoints included OS, event-free survival (EFS) and relapse-free survival (RFS), non-hematological toxicities and hematological toxicities during induction, duration of critical cytopenia, and duration of hospitalization. These secondary endpoints were exploratory in nature and were not powered to be definitive. 
Table 1 Patient characteristics by randomization

\begin{tabular}{|c|c|c|c|}
\hline \multirow[t]{2}{*}{ Treatment arm } & \multicolumn{3}{|c|}{$\begin{array}{l}\text { All randomized and evaluable patients in the } \\
\text { study }\end{array}$} \\
\hline & Standard & S-HAM & $P$ \\
\hline No. of patients, $N(\%)$ & $184(48)$ & $203(52)$ & \\
\hline Age, years & & & $0.722^{\mathrm{a}}$ \\
\hline Median (range) & $58(18-86)$ & $58(19-81)$ & \\
\hline Sex & & & $0.026^{\mathrm{b}}$ \\
\hline Female/male, $N(\%)$ & $76 / 108(41 / 59)$ & $107 / 96(53 / 47)$ & \\
\hline $\begin{array}{l}\text { Patient age } \\
\text { (chronological), } N(\%)\end{array}$ & & & $0.760^{\mathrm{b}}$ \\
\hline$<60$ years & $101(55)$ & $108(53)$ & \\
\hline$\geq 60$ years & $83(45)$ & $95(47)$ & \\
\hline \multicolumn{4}{|l|}{$\begin{array}{l}\text { Patient age (biological), } \\
N(\%)\end{array}$} \\
\hline Younger: $<60(-70)$ & $119(65)$ & $132(65)$ & \\
\hline Median (range) & $51(18-68)$ & $52(19-70)$ & $0.815^{\mathrm{a}}$ \\
\hline Older: $\geq 60(-70)$ & $65(35)$ & $71(35)$ & \\
\hline Median (range) & $68(60-86)$ & $68(57-81)$ & $0.101^{\mathrm{a}}$ \\
\hline AML subtype, $N(\%)$ & & & $0.091^{\mathrm{b}}$ \\
\hline de novo AML & $143(78)$ & $154(76)$ & \\
\hline $\begin{array}{l}\text { AML secondary to } \\
\text { MDS (s-AML) }\end{array}$ & $22(12)$ & $37(18)$ & \\
\hline $\begin{array}{l}\text { AML therapy-related (t- } \\
\text { AML) }\end{array}$ & $19(10)$ & $12(6)$ & \\
\hline Blasts in bone marrow, $\%$ & & & $0.551^{\mathrm{a}}$ \\
\hline Median & 65 & 68 & \\
\hline Range & $10-97$ & $6-100$ & \\
\hline $\mathrm{LDH}, \mathrm{U} / \mathrm{L}$ & & & $0.721^{\mathrm{a}}$ \\
\hline Median (range) & $334(107-4833)$ & $356(117-3431)$ & \\
\hline ECOG, $N(\%)$ & & & $0.631^{\mathrm{b}}$ \\
\hline 0 & $65(36)$ & $62(31)$ & \\
\hline 1 & $94(52)$ & $107(53)$ & \\
\hline 2 & $17(9)$ & $23(11)$ & \\
\hline 3 & $5(3)$ & $7(4)$ & \\
\hline 4 & 0 & $2(1)$ & \\
\hline Missing/unknown & 3 & 2 & \\
\hline Karyotype, $N(\%)$ & & & $0.006^{\mathrm{b}}$ \\
\hline Favorable & $15(9)$ & $5(3)$ & \\
\hline Intermediate & $104(65)$ & $146(78)$ & \\
\hline Unfavorable & $41(26)$ & $37(20)$ & \\
\hline Missing/unknown & 24 & 15 & \\
\hline $\begin{array}{l}\text { Molecular aberrations, } \\
N(\%)\end{array}$ & & & $0.178^{\mathrm{b}}$ \\
\hline \multicolumn{4}{|l|}{ NPM1 } \\
\hline Pos. & $53(33)$ & $74(41)$ & \\
\hline Neg. & $106(67)$ & $107(59)$ & \\
\hline Missing/unknown & 25 & 22 & \\
\hline FLT3-ITD & & & $0.895^{\mathrm{b}}$ \\
\hline Pos. & $34(21)$ & $38(21)$ & \\
\hline Neg. & $125(79)$ & $146(79)$ & \\
\hline Missing/unknown & 25 & 19 & \\
\hline FLT3-TKD & & & $1.000^{\mathrm{b}}$ \\
\hline Pos. & $11(8)$ & $10(8)$ & \\
\hline Neg. & $120(92)$ & $121(92)$ & \\
\hline Unknown & 53 & 72 & \\
\hline MLL-PTD & & & $0.554^{\mathrm{b}}$ \\
\hline
\end{tabular}

Table 1 (continued)

\begin{tabular}{llll}
\hline Treatment arm & \multicolumn{3}{l}{$\begin{array}{l}\text { All randomized and evaluable patients in the } \\
\text { study }\end{array}$} \\
\cline { 2 - 4 } & Standard & S-HAM & $P$ \\
\hline Pos. & $11(7)$ & $17(9)$ & \\
Neg. & $143(93)$ & $164(91)$ & \\
Missing/unknown & 30 & 22 & $0.676^{\mathrm{b}}$ \\
CEBPA & & & \\
Pos. & $4(11)$ & $2(6)$ & \\
Neg. & $32(89)$ & $30(94)$ & \\
Missing/unknown & 148 & 171 & \\
\hline
\end{tabular}

$M D S$ myelodysplastic syndrome, $L D H$ lactate dehydrogenase serum level, ECOG Eastern Cooperative Oncology Group, NPM1 nucleophosmin, FLT3 fms-like tyrosine kinase 3, ITD internal tandem duplication, TKD tyrosine kinase domain, MLL-PTD mixed linage leukemia-partial tandem duplication, CEBPA CCAAT/enhancer-binding protein alpha

${ }^{\text {a }} P$-values are from Wilcoxon rank-sum test

${ }^{\mathrm{b}} P$-values are from Fisher's exact test

\section{Statistics}

The trial addressed the hypothesis that the ORR might be improved by S-HAM by $15 \%$ from $70 \%$ as expected for standard double induction to $85 \%$ for S-HAM. The primary confirmatory endpoint was analyzed using a sequential onesided truncated probability ratio test [14]. The significance level was set to 0.05 . Based on these assumptions, 360 evaluable patients had to be recruited to achieve a power of 95\% (see study protocol). Patient characteristics between the two randomized treatment regimens were evaluated using Fisher's exact test for categorical and Wilcoxon ranksum test for continuous variables with the respective $95 \%$ confidence intervals $(95 \% \mathrm{CI})$. Categorical variables are reported as absolute and relative frequencies. Continuous variables are shown as median [minimum-maximum]. Time-to-event outcomes were analyzed by two-sided logrank tests. Kaplan-Meier method was used to estimate event rates and 95\% confidence limits (95\% CI). Duration of critical cytopenia was calculated from the start of therapy until recovery of peripheral blood counts and was presented as inverse Kaplan-Meier curves. Primary and secondary efficacy analyses were performed on an intention-to-treat basis. All secondary and subgroup analyses have to be considered exploratory and thus no adjustment for multiplicity was performed. $P$-values $\leq 0.05$ were considered as significant. The primary analysis (sequential test) was performed using the SAS software $\left(\mathrm{SAS}^{\circledR}\right.$ software, version 9.2, for Windows (SAS Institute, Cary, NC, USA)). All other statistical analyses were performed using the $\mathrm{SAS}^{\circ}$ software version 9.4. 
Table 2 Treatment outcome by randomization

\begin{tabular}{|c|c|c|c|c|c|c|c|}
\hline \multirow[t]{3}{*}{ Treatment arm } & \multicolumn{7}{|c|}{ All randomized and evaluable patients in the study } \\
\hline & \multicolumn{2}{|c|}{ Total patients } & \multicolumn{2}{|l|}{ Standard ${ }^{\mathrm{a}}$} & \multicolumn{3}{|l|}{ S-HAM } \\
\hline & $N(\%)$ & $95 \% \mathrm{CI}$ & $N(\%)$ & $95 \% \mathrm{CI}$ & $N(\%)$ & $95 \% \mathrm{CI}$ & $P$ \\
\hline \multicolumn{8}{|l|}{ Patients randomized } \\
\hline Total & 387 & & 184 & & 203 & & \\
\hline Younger & 251 & & 119 & & 132 & & \\
\hline Older & 136 & & 65 & & 71 & & \\
\hline \multicolumn{8}{|c|}{ Overall response $(\mathrm{CR}+\mathrm{CRi})$} \\
\hline Total & $289(75)$ & $70-79$ & $133(72)$ & $65-79$ & $156(77)$ & $70-83$ & $0.202^{\mathrm{b}}$ \\
\hline Younger & $197(79)$ & $73-83$ & $91(76)$ & $68-83$ & $106(80)$ & $73-86$ & $0.539^{\mathrm{c}}$ \\
\hline Older & $92(68)$ & $59-75$ & $42(65)$ & $53-75$ & $50(70)$ & $59-80$ & $0.582^{\mathrm{c}}$ \\
\hline \multicolumn{8}{|l|}{ Induction result } \\
\hline Total & & & & & & & $0.309^{\mathrm{c}}$ \\
\hline $\mathrm{CR}$ & $200(52)$ & $46-57$ & $86(47)$ & $39-54$ & $114(56)$ & $49-63$ & \\
\hline CRi & $89(23)$ & $19-28$ & $47(25)$ & $19-32$ & $42(21)$ & $15-27$ & \\
\hline Persistent leukemia & $48(12)$ & $9-16$ & $24(13)$ & $8-19$ & $24(12)$ & $7-17$ & \\
\hline Early death & $50(13)$ & $9-17$ & $27(15)$ & $9-21$ & $23(11)$ & $7-17$ & \\
\hline Younger & & & & & & & $0.717^{\mathrm{c}}$ \\
\hline $\mathrm{CR}$ & $136(54)$ & $47-60$ & $60(50)$ & $41-60$ & $76(57)$ & $48-66$ & \\
\hline $\mathrm{CRi}$ & $61(24)$ & $19-30$ & $31(26)$ & $18-35$ & $30(23)$ & $16-31$ & \\
\hline Persistent leukemia & $24(10)$ & $6-14$ & $12(10)$ & $6-17$ & $12(9)$ & $5-15$ & \\
\hline Early death & $30(12)$ & $8-17$ & $16(14)$ & $8-21$ & $14(11)$ & $6-17$ & \\
\hline Older & & & & & & & $0.438^{\mathrm{c}}$ \\
\hline $\mathrm{CR}$ & $64(47)$ & $38-56$ & $26(40)$ & $28-53$ & $38(53)$ & $41-65$ & \\
\hline $\mathrm{CRi}$ & $28(20)$ & $14-28$ & $16(25)$ & $15-37$ & $12(17)$ & $9-28$ & \\
\hline Persistent leukemia & $24(18)$ & $12-25$ & $12(18)$ & $10-30$ & $12(17)$ & $9-27$ & \\
\hline Early death & $20(15)$ & $9-22$ & $11(17)$ & $8-28$ & $9(13)$ & $6-23$ & \\
\hline \multicolumn{8}{|l|}{ Early death until day 90} \\
\hline Total & $60(16)$ & $12-20$ & $29(16)$ & $11-22$ & $31(15)$ & $11-21$ & $1.000^{\mathrm{c}}$ \\
\hline Younger & $36(14)$ & $11-19$ & $16(13)$ & $8-21$ & $20(15)$ & $10-22$ & $0.722^{\mathrm{c}}$ \\
\hline Older & $24(18)$ & $12-25$ & $13(20)$ & $12-31$ & $11(16)$ & $9-26$ & $0.509^{c}$ \\
\hline \multicolumn{8}{|l|}{ Overall survival } \\
\hline Total & & & & & & & $0.323^{\mathrm{d}}$ \\
\hline Median, months & 29 & $20-38$ & 25 & $15-34$ & 35 & $21-49$ & \\
\hline Younger & & & & & & & $0.742^{\mathrm{d}}$ \\
\hline Median, months & 48 & $25-71$ & 45 & $16-74$ & 48 & $25-71$ & \\
\hline Older & & & & & & & $0.219^{\mathrm{d}}$ \\
\hline Median, months & 19 & $12-25$ & 19 & $10-27$ & 19 & $11-28$ & \\
\hline \multicolumn{8}{|l|}{ Event-free survival } \\
\hline Total & & & & & & & $0.753^{\mathrm{d}}$ \\
\hline Median, months & 11 & $8-13$ & 10 & $6-15$ & 11 & $8-14$ & \\
\hline Younger & & & & & & & $0.980^{\mathrm{d}}$ \\
\hline Median, months & 14 & $7-22$ & 18 & $7-29$ & 14 & $6-21$ & \\
\hline Older & & & & & & & $0.441^{\mathrm{d}}$ \\
\hline Median, months & 7 & $4-10$ & 6 & $3-9$ & 9 & $4-14$ & \\
\hline
\end{tabular}

CR complete remission, $C R i$ incomplete remission

aStandard treatment in "younger" patients was TAD-HAM (double induction mandatory), in "older" patients it was HAM(-HAM) (double induction only conditional if no adequate blast clearance ( $5 \%$ ) had been achieved after one cycle of HAM)

${ }^{\mathrm{b}} P$-values are from one-sided sequential truncated probability ratio test (Whitehead)

${ }^{\mathrm{c}} P$-values are from Fisher's exact test

${ }^{\mathrm{d}} P$-values are from log-rank test 


\section{Results}

\section{Patient characteristics}

From July 2009 until March 2012, a total of 396 patients were randomized into the study. After exclusion of nine patients, 387 evaluable patients were compared between S-HAM $[n=203(52 \%)]$ and standard double induction [n=184 (48\%)] (Fig. 2). Patient characteristics were similar between both treatment groups (Table 1). The median age was 58 years, $46 \%$ of patients had a chronological age $\geq 60$ years. The group of "younger" patients including patients between 60 and 70 years who were considered biologically young consisted of 251 (65\%) patients and was randomized between S-HAM and (mandatory) DI with TAD-HAM. Correspondingly, 136 $(35 \%)$ patients belonged to the "older" age group $\geq 60$ and were randomized between S-HAM and (conditional) DI with HAM(-HAM). Compliance to the assigned therapy was $99 \%$ in both the treatment groups.

\section{Treatment response $\left(1^{\circ}\right.$ endpoint) and survival $\left(2^{\circ}\right.$ endpoint)}

In the total group, the ORR (consisting of $\mathrm{CR}$ and $\mathrm{CR}_{\mathrm{i}}$ ) was $75 \%$, the rate of persistent leukemia (PL) was $12 \%$, and the rate of early death (ED) was $13 \%$. ED until day 90 was $16 \%$ (Table 2). Please note that some patients had PL as their induction result but then died later on but within 90 days after the start of treatment. These patients were also counted in the "ED until day 90".

In the S-HAM group, ORR was higher but not statistically significant with $77 \%$ (95\% CI: 70-83\%) as compared to $72 \%$ (95\% CI: $65-79 \%)$ for the standard DI group $(P=0.202)$. In the "younger" group the ORR after S-HAM was $80 \%$ versus $76 \%$ after standard DI with TAD-HAM. In the "older" group the ORR after S-HAM was $70 \%$ versus $65 \%$ after standard DI with HAM(-HAM).

The median follow-up was 62 months. Over all patients the median OS was 29 months. Following S-HAM treatment, the median OS was 35 months versus 25 months after standard DI $(P=0.323)$. For the age strata, the results on OS were 48 months for S-HAM versus 45 months after standard DI in the "younger" group and 19 months (S-HAM) versus 19 months (standard DI) in the "older" group (Fig. 3). There were no significant differences in EFS (also Fig. 3) and in RFS (data not shown).

No significant differences between treatment arms were observed for ORR nor OS in the prespecified karyotype subgroups (data not shown).
Toxicities and early death (ED) rate ( $2^{\circ}$ endpoints)

Non-hematological toxicities grade 3 and 4 are listed in Table 3. For the total group, the most relevant toxicities were infection $48 \%$, pulmonary toxicity $19 \%$, pain $16 \%$, fever $13 \%$, weight gain $12 \%$, diarrhea $11 \%$, liver toxicity $10 \%$, nausea/vomiting $9 \%$, and bleeding $7 \%$. There were no statistically significant differences between the S-HAM group and the standard DI group except for bleeding, 10\% (S-HAM) versus $4 \%$ (standard DI) and mucositis, 10\% (S-HAM) versus 3\% (standard DI).

The rate of ED was evaluated for the following time periods: days $1-14\left(\mathrm{ED}_{1-14}\right) 3 \%$, days $1-30\left(\mathrm{ED}_{1-30}\right) 7 \%$, days $1-60\left(\mathrm{ED}_{1-60}\right) 12 \%$, and days $1-90\left(\mathrm{ED}_{1-90}\right) 16 \%$. There were no statistically significant differences between the S-HAM arm $\left(\mathrm{ED}_{1-14}: 4 \%, \mathrm{ED}_{1-30}: 7 \%, \mathrm{ED}_{1-}\right.$ $\left.{ }_{60}: 12 \%, \mathrm{ED}_{1-90}: 15 \%\right)$ and the standard DI arm $\left(\mathrm{ED}_{1-14}:\right.$ $\left.2 \%, \quad \mathrm{ED}_{1-30}: \quad 6 \%, \mathrm{ED}_{1-60}: 13 \%, \mathrm{ED}_{1-90}: 16 \%\right)$, respectively.

\section{Duration of critical cytopenias ( $2^{\circ}$ endpoints)}

After S-HAM, the median duration of critical leukopenia (until recovery to $\geq 1.000 / \mu l$ leukocytes) was significantly shorter with 29 days versus 44 days after standard DI $(P$ $<0.001)$-Fig. 4. Within the age strata, we found critical leukopenia of 29 days after S-HAM versus 46 days after standard DI in "younger" patients $(P<0.001)$ and 27 days versus 51 days in "older" patients, if the patients had received two cycles of HAM. Similarly, the median duration of critical thrombocytopenia (46 days versus 33 days) and neutropenia (50 days versus 31 days) were also significantly reduced after S-HAM as compared to standard DI (Supplementary Figures 1 and 2). If "older" patients in the control arm had received only one cycle of therapy (=positive selection of patients) then there was no further shortening of cytopenias in the experimental arm after S-HAM (Fig. 4d, Supplementary Figures 1D, 2D).

\section{Duration of hospitalization ( $2^{\circ}$ endpoint)}

For the whole group, the median duration of hospitalization (counted from day 1 of study treatment to the day of hospital discharge) was significantly shorter after S-HAM with 37 days versus 49 days after standard DI $(P<0.001)$ Fig. 5. The respective data were 37 days versus 50 days after standard DI in "younger" patients $(P<0.001)$ and 35 days versus 57 days in "older" patients, if the patients had received two cycles of HAM. 


\section{A}

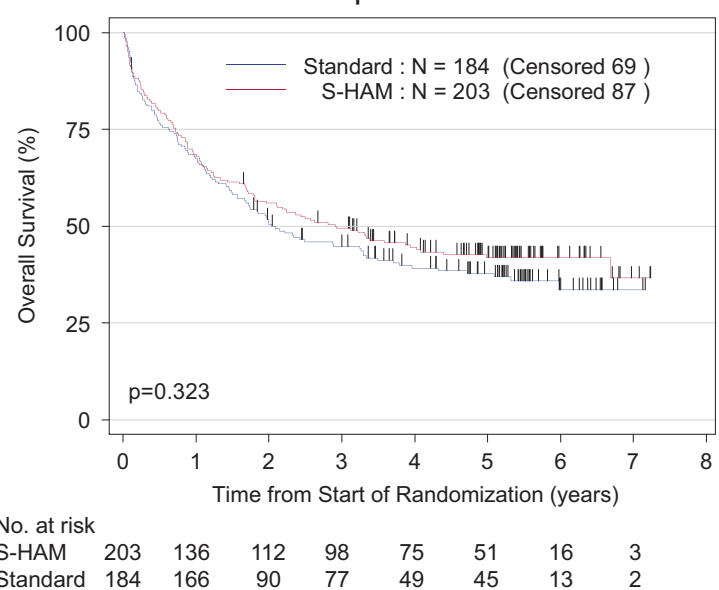

B

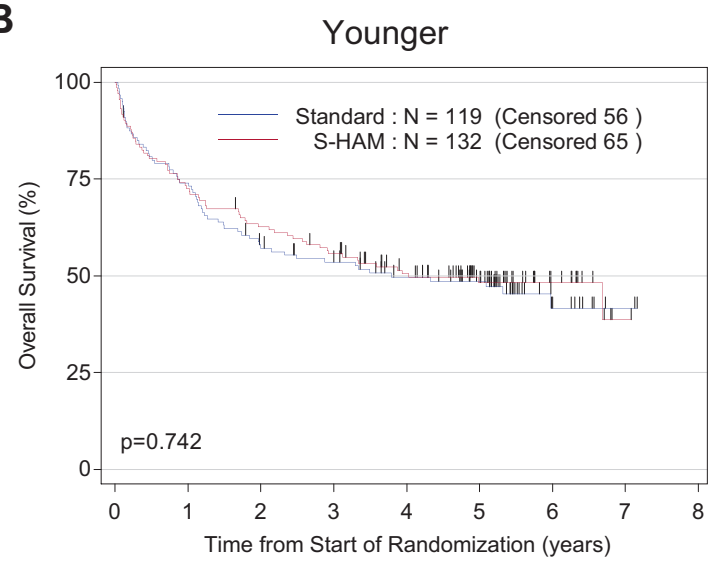

No. at risk

$\begin{array}{lllllllll}\text { S-HAM } & 132 & 94 & 81 & 68 & 39 & 19 & 2 & 1 \\ \text { Standard } & 119 & 86 & 64 & 56 & 34 & 13 & 2 & 0\end{array}$

\section{C}

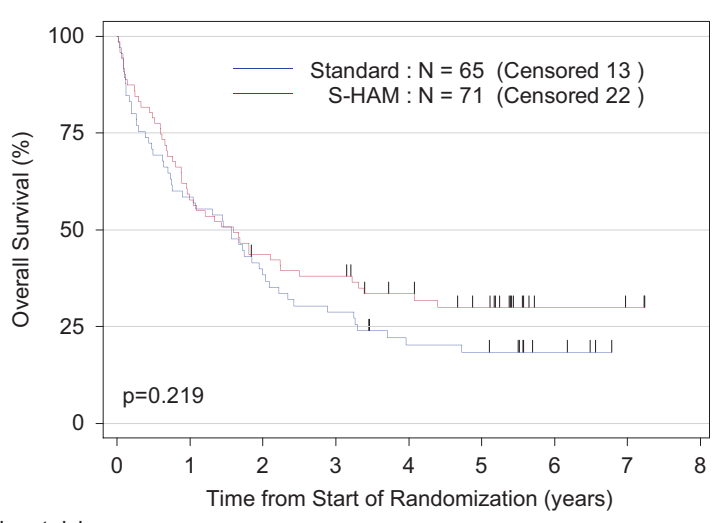

No. at risk

$\begin{array}{lllllllll}\text { S-HAM } & 71 & 41 & 31 & 27 & 20 & 15 & 3 & 2\end{array}$

Fig. 3 Overall survival and event-free survival. Overall survival of all patients (a), of patients younger than $<60$ years $(\mathbf{b})$, of patients older than $\geq 60$ years $(\mathbf{c})$. Event-free survival of all patients $(\mathbf{d})$, of patients
D

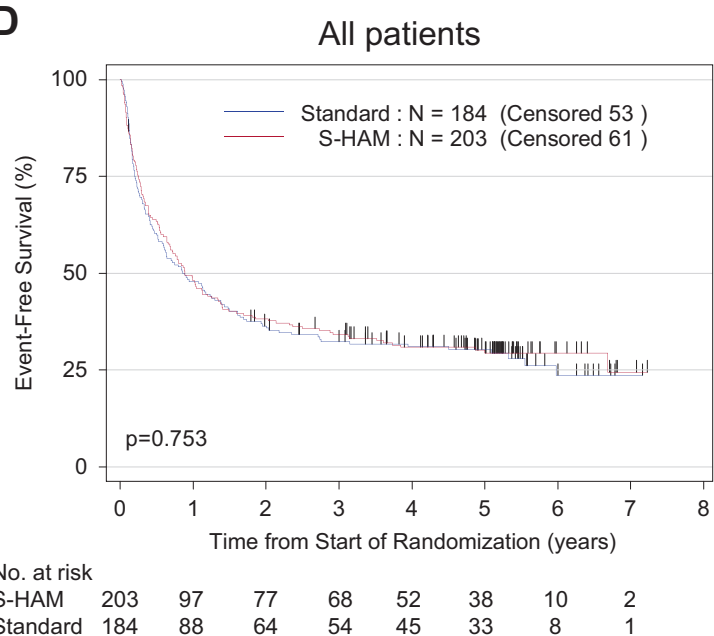

$\mathbf{E}$

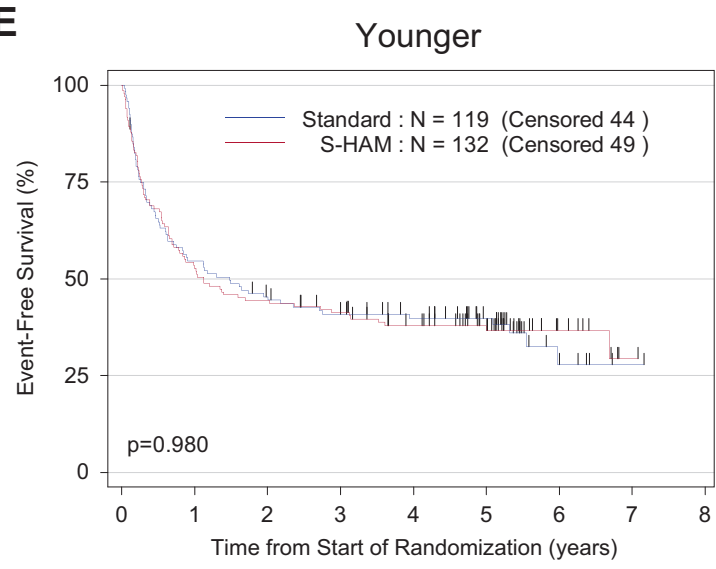

No. at risk

$\begin{array}{lllllllll}\text { S-HAM } & 132 & 69 & 58 & 53 & 42 & 29 & 9 & 1 \\ \text { Standard } & 119 & 65 & 52 & 44 & 37 & 26 & 5 & 1\end{array}$

$\mathbf{F}$

Older

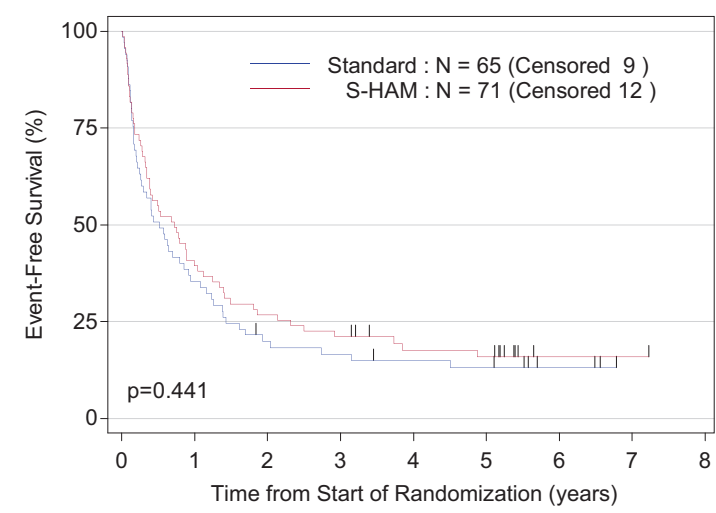

No. at risk

$\begin{array}{lllllclll}\text { S-HAM } & 71 & 28 & 19 & 15 & 10 & 9 & 1 & 1 \\ \text { Standard } & 65 & 23 & 12 & 10 & 8 & 7 & 3 & 0\end{array}$

younger than $<60$ years $(\mathbf{e})$, of patients older than $\geq 60$ years (f) always biological age as defined in "Patients and methods" 
Table 3 Incidence of non-hematological toxicities grade 3 and 4 by randomization

\begin{tabular}{|c|c|c|c|c|c|c|}
\hline \multirow[t]{3}{*}{ Treatment arm } & \multicolumn{6}{|c|}{ All randomized and evaluable patients in the study } \\
\hline & \multirow{2}{*}{\multicolumn{2}{|c|}{$\frac{\text { Total patients }}{n / N(\%)}$}} & \multirow{2}{*}{\multicolumn{2}{|c|}{$\frac{\text { Standard }}{n / N(\%)}$}} & \multirow{2}{*}{\multicolumn{2}{|c|}{$\frac{\text { S-HAM }}{n / N(\%)}$}} \\
\hline & & & & & & \\
\hline Alopecia & $141 / 259$ & $(54.4)$ & $70 / 132$ & $(53.0)$ & $71 / 127$ & $(56.0$ \\
\hline Infection & $166 / 349$ & $(47.6)$ & $80 / 181$ & $(44.2)$ & $86 / 168$ & $(51.2$ \\
\hline $\begin{array}{l}\text { Pulmonary } \\
\text { toxicity }\end{array}$ & $66 / 352$ & $(18.8)$ & $28 / 181$ & $(15.5)$ & $38 / 171$ & $(22.2$ \\
\hline Prothrombin & $17 / 281$ & (6.0) & $6 / 149$ & $(4.0)$ & $11 / 132$ & $(8.3)$ \\
\hline Fever & $45 / 353$ & $(12.7)$ & $26 / 183$ & $(14.2)$ & $19 / 170$ & $(11.2$ \\
\hline Diarrhea & $39 / 352$ & $(11.1)$ & $17 / 181$ & $(9.4)$ & $22 / 171$ & $(12.9$ \\
\hline Pain & $55 / 351$ & $(15.7)$ & $22 / 180$ & (12.2) & $33 / 171$ & $(19.3$ \\
\hline Nausea/vomiting & $31 / 351$ & (8.8) & $16 / 180$ & (8.9) & $15 / 171$ & $(8.8)$ \\
\hline PCHE & $3 / 209$ & (1.4) & $1 / 110$ & $(0.9)$ & $2 / 99$ & $(2.0)$ \\
\hline Hypoproteinemia & $30 / 296$ & $(10.1)$ & $15 / 151$ & $(9.9)$ & $15 / 145$ & $(10.3$ \\
\hline Mucositis & $22 / 344$ & (6.4) & $5 / 177$ & (2.8) & $17 / 167$ & $(10.2$ \\
\hline Weight gain & $43 / 352$ & (12.2) & $22 / 183$ & (12.0) & $21 / 169$ & $(12.4$ \\
\hline Cardiac function & $12 / 346$ & (3.5) & $8 / 179$ & $(4.5)$ & $4 / 167$ & (2.4) \\
\hline Bleeding & $25 / 350$ & (7.1) & $8 / 183$ & (4.4) & $17 / 167$ & $(10.2$ \\
\hline SGOT/SGPT & $37 / 351$ & $(10.5)$ & $18 / 180$ & (10.0) & $19 / 171$ & $(11.1$ \\
\hline $\begin{array}{l}\text { Central nervous } \\
\text { system }\end{array}$ & $18 / 349$ & (5.2) & $5 / 179$ & (2.8) & $13 / 170$ & (7.7) \\
\hline Bilirubin & $16 / 352$ & (4.5) & $6 / 181$ & (3.3) & $10 / 171$ & $(5.9)$ \\
\hline Effusion & $17 / 350$ & (4.9) & $8 / 180$ & (4.4) & $9 / 170$ & (5.3) \\
\hline Edema & $21 / 350$ & (6.0) & $8 / 179$ & $(4.5)$ & $13 / 171$ & (7.6) \\
\hline $\begin{array}{l}\text { Cutaneous } \\
\text { toxicity }\end{array}$ & $8 / 348$ & (2.3) & $2 / 181$ & (1.1) & $6 / 167$ & (3.6) \\
\hline $\begin{array}{l}\text { Alkaline } \\
\text { phosphatase }\end{array}$ & $4 / 306$ & (1.3) & $2 / 156$ & (1.3) & $2 / 150$ & (1.3) \\
\hline Cardiac rhythm & $7 / 344$ & $(2.0)$ & $3 / 180$ & (1.7) & $4 / 164$ & (2.4) \\
\hline $\begin{array}{l}\text { Creatinine/renal } \\
\text { toxicity }\end{array}$ & $3 / 352$ & $(0.9)$ & $3 / 181$ & (1.7) & $0 / 171$ & $(0.0)$ \\
\hline Obstipation & $5 / 351$ & (1.4) & $2 / 181$ & (1.1) & $3 / 170$ & (1.8) \\
\hline Hematuria & $1 / 347$ & $(0.3)$ & $1 / 179$ & $(0.6)$ & $0 / 168$ & $(0.0)$ \\
\hline Extrapyramidal & $4 / 350$ & (1.1) & $2 / 180$ & (1.1) & $2 / 170$ & (1.2) \\
\hline Allergic reaction & $2 / 346$ & $(0.6)$ & $1 / 181$ & $(0.6)$ & $1 / 165$ & (0.6) \\
\hline $\begin{array}{l}\text { Peripheral } \\
\text { nervous system }\end{array}$ & $3 / 351$ & $(0.9)$ & $2 / 181$ & (1.1) & $1 / 170$ & $(0.6)$ \\
\hline Pericarditis & $0 / 346$ & $(0.0)$ & $0 / 178$ & $(0.0)$ & $0 / 168$ & $(0.0)$ \\
\hline
\end{tabular}

$P C H E$ pseudocholinesterase, SGOT/SGPT serum-glutamat-oxalacetattransaminase/serum-glutamat-pyruvat-transferase

\section{Conclusion}

The current study evaluated whether shortening of induction therapy by the dose-dense S-HAM regimen might improve the response rate and overall prognosis of patients with newly diagnosed AML. This approach was based on promising results of a preceding trial in relapsed and refractory AML and a pilot study in previously untreated patients. The observed results revealed only a trend with a $5 \%$ higher ORR of $77 \%$ versus $72 \%$ after standard induction therapy, which was not statistically significant. This applied both for the younger group, where double induction is mandatory as well as for the older group, where double induction is only conditional-i.e., the second cycle is only applied if there were residual blasts on the day 16 bone marrow aspirate. Similarly, there was only a trend towards a longer OS with 35 months after S-HAM versus 25 months after standard DI $(P=0.323)$. No differences between both strategies were observed for the ED rate.

However, a clinically relevant and statistically significant difference was found for the duration of critical cytopenias in favor of S-HAM. Especially, critical leukopenia was reduced by a delta of 15 days from 44 days in the standard arm to 29 days after S-HAM. We attribute this phenomenon primarily to the "hematopoiesis protective timing" of treatment days $8-11$ of S-HAM. This early treatment avoids the myelosuppressive effect of standard double induction, where the second cycle is started on day 21 , when normal hematopoiesis is usually just starting to regenerate. The use of G-CSF following antileukemic therapy has been shown to reduce leukopenia by a median of 4 days after S-HAM induction as well as after other regimens such as high-dose AraC consolidation therapy $[15,16]$. In relation to the total delta of 15 days, the differential use of G-CSF is therefore only a minor contributor. This is especially true because even though G-CSF use was mandatory after S-HAM and only optional after standard DI—nevertheless a substantial proportion of standard DI patients (17\%) also received GCSF thus making the component of differential G-CSF use even less relevant.

Even though this shortened leukopenia after S-HAM was not associated with a reduction in toxicities or in the ED rate, it nevertheless allowed a substantially earlier discharge of patients out of the hospital. The median duration of hospitalization was significantly reduced from 7 weeks (49 days) to 5 weeks (37 days). Even though these secondary endpoints were exploratory in nature and not powered to be definitive, they nevertheless precisely replicate the findings of our pilot study [10].

Our study should be considered in the context of other studies that have evaluated a "dose-dense" or "timesequential" approach in which two cycles of intensive chemotherapy were applied during induction with either a "standard interval"-i.e., the 2 nd cycle starting on day $21-$ or a substantially shorter interval between the cycles ("dosedense" approach). The most convincing data were shown for pediatric AML patients in the CCG-2891 study by the Childrens' Oncology Group COG [17] where two cycles of the DCTER regimen (Dexamethasone, Cytarabine, 

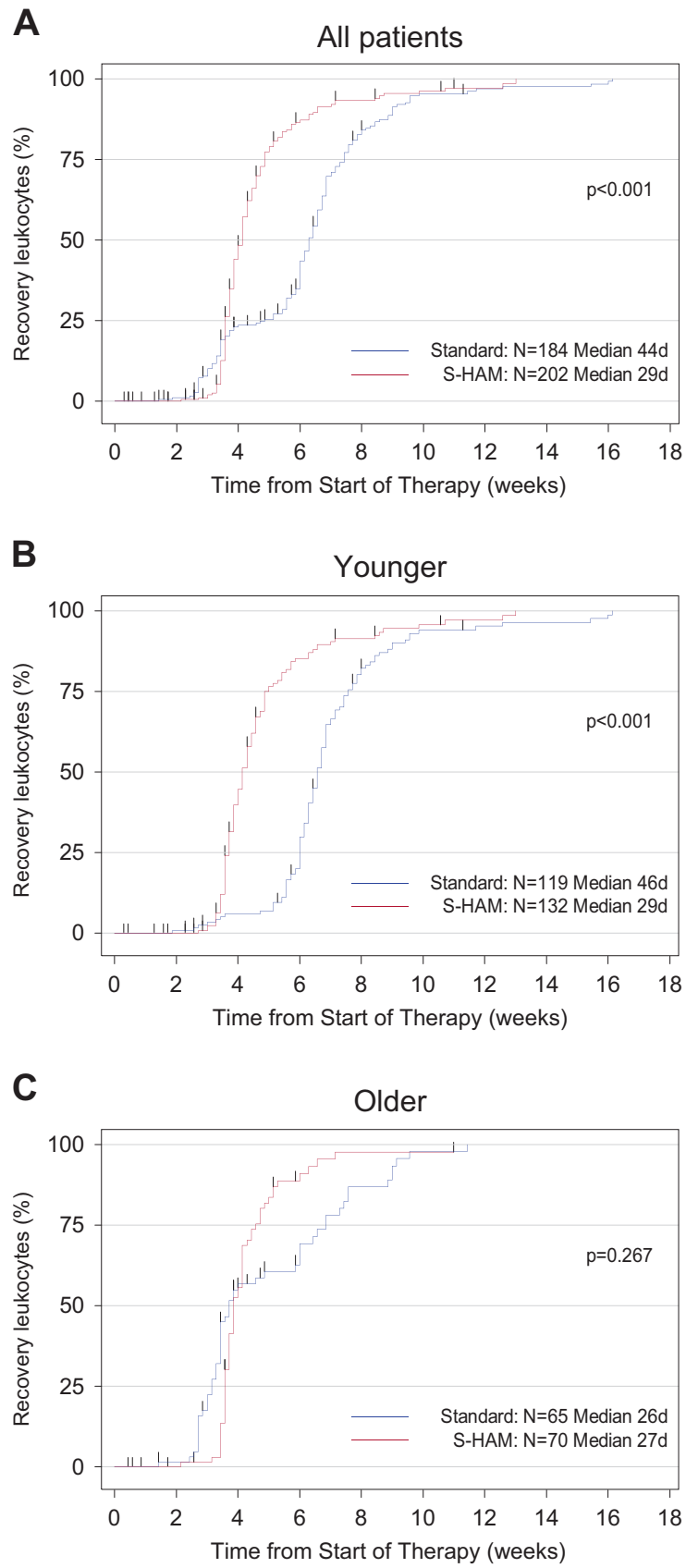

Fig. 4 Duration of leukopenia. Duration of critical leukopenia $(<1.000$ leukocytes/ $\mu \mathrm{l}$ ) in all patients (a), in patients younger than $<60$ years (b), in patients older than $\geq 60$ years (c). Duration of critical leukopenia was calculated from the start of therapy until recovery of peripheral blood counts and was presented as inverse Kaplan-Meier curves. In (c) please note the "bump" in the standard group (blue line), which is due to the fact that one subgroup of patients received only one cycle of HAM (positive selection because of adequate blast clearance in the day 16 bone marrow aspirate) and the other subgroup received two cycles

Thioguanine, Etoposide, Daunorubicine) were applied. After dose-dense application with a 6-day interval between cycles as compared to the 2nd cycle given only after hematologic regeneration, no increase in the $\mathrm{CR}$ rate was noted but long-term follow-up demonstrated a substantial
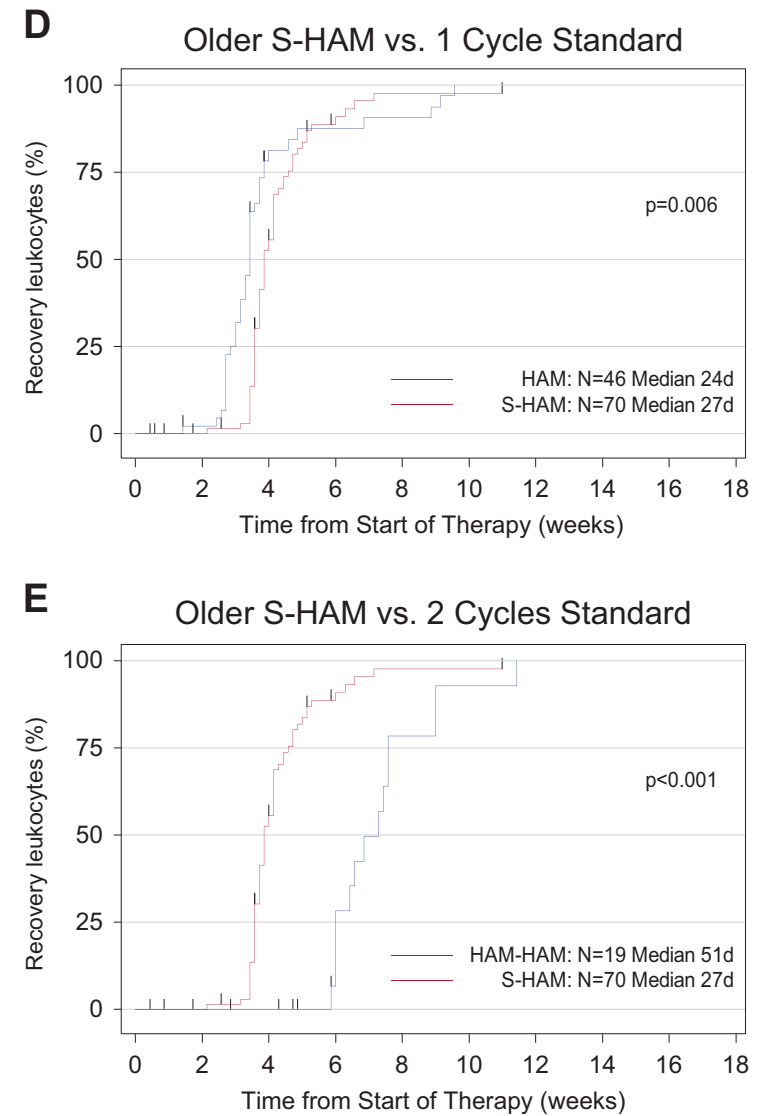

of HAM (negative selection because of residual blasts in the day 16 bone marrow aspirate). Comparison of the duration of critical leukopenia $(<1.000$ leukocytes/ $\mu \mathrm{l})$ of all S-HAM patients older than $\geq 60$ years versus those standard arm patients who received only one cycle of HAM (positive selection because of adequate blast clearance in the day 16 bone marrow aspirate) (d), of all S-HAM patients older than $\geq 60$ years versus those standard arm patients who received two cycles of HAM (negative selection because of residual blasts in the day 16 bone marrow aspirate) (e)

prolongation of EFS and OS [18]. In adult patients, the French ALFA 9000 study [7] compared two cycles of intensive chemotherapy with the 2nd cycle given after a 4day interval on day 8 (dose-dense approach) and a standard arm with the 2 nd cycle given on day 20 . In this study, only 
A
All patients

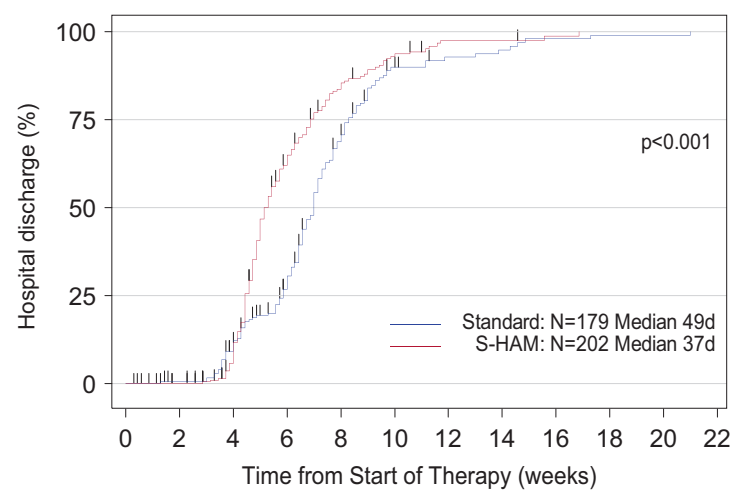

B

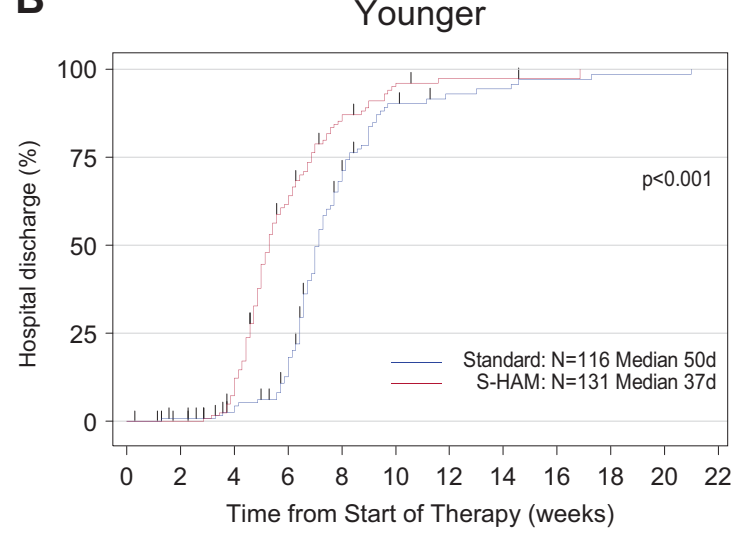

C

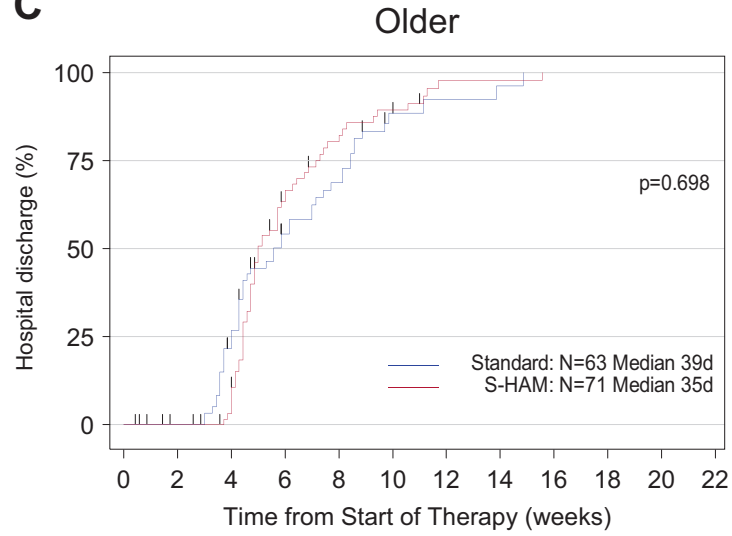

Fig. 5 Duration of hospitalization. Duration of hospitalization in all patients (a), in patients younger than $<60$ years (b), in patients older than $\geq 60$ years $(\mathbf{c})$. Duration of hospitalization was calculated from the start of therapy until the day of discharge and was presented as inverse Kaplan-Meier curves. In (c) please note the "bump" in the standard group (blue line), which is due to the fact that one subgroup of patients received only one cycle of HAM (positive selection because of adequate blast clearance in the day 16 bone marrow aspirate) and the other subgroup received two cycles of HAM (negative selection because of

a difference in the relapse-free interval in favor of the dosedense approach was seen. No differences were seen for the $\mathrm{CR}$ rate, EFS, and OS.

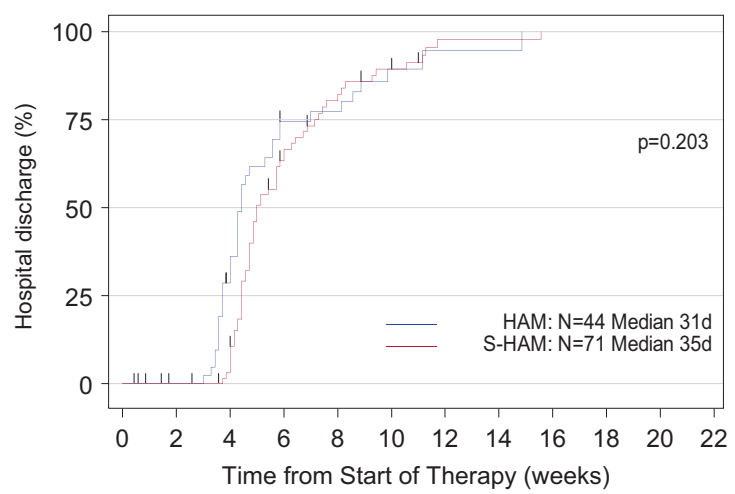

E

Older S-HAM vs. 2 Cycles Standard

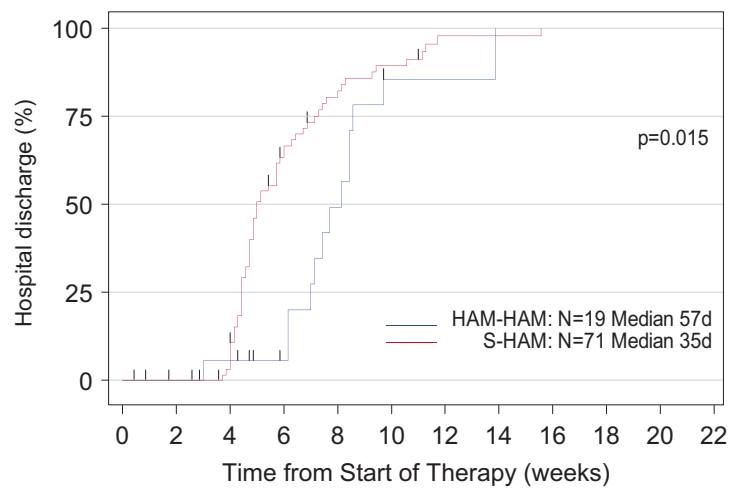

D Older S-HAM vs. 1 Cycle Standard

residual blasts in the day 16 bone marrow aspirate). Comparison of the duration of hospitalization of all S-HAM patients older than $\geq 60$ years versus those standard arm patients who received only one cycle of HAM (positive selection because of adequate blast clearance in the day 16 bone marrow aspirate) (d), of all S-HAM patients older than $\geq 60$ years versus those standard arm patients who received two cycles of HAM (negative selection because of residual blasts in the day 16 bone marrow aspirate) (e)

Our study has several limitations: (1) the hypothesis that S-HAM might improve ORR by $15 \%$ as compared to standard double induction might have been too ambitious. 
Hence, the number of patients recruited into the trial did not allow demonstrating a smaller though clinically relevant difference in ORR. (2) Curative AML (induction) treatment is not standardized and the regimens that we used in our study are-even though used frequently-not necessarily representative of AML induction regimens used in other parts of the world or in different study groups. (3) Genotype-specific therapy for some subgroups of AML, especially those with FLT3 mutations, is now successfully combined with intensive chemotherapy backbones $[2,19]$. In how far these new agents that were used in combination with "7+3" type induction can be combined with other chemotherapy backbones like S-HAM needs to be clarified. (4) The substantially shortened duration of leukopenia and the resulting shortening of hospitalization make the S-HAM regimen an attractive option as a feasible and reliable chemotherapy backbone during induction. However, we must consider that the shortened leukopenia is pronounced only when compared to mandatory DI. Even though mandatory DI is used in many regions of the world and in many study groups $[5-8,11]$, its superiority to conditional DI has only been shown in historical comparisons [5, 18]. However, this question will be answered in a prospective randomized comparison by the "Dauno Double" Study of the German SAL study group which is presently recruiting.

In conclusion, the S-HAM regimen demonstrates favorable trends but no significant differences in ORR and OS as compared to standard DI. The regimen shortens critical leukopenia by more than 2 weeks with a subsequent reduction in the lengths of hospitalization from 7 to 5 weeks. In our opinion, this shorter hospital stay will appeal to patients and should also be financially favorable in most health care systems. Altogether, these features make the S-HAM regimen an attractive chemotherapy backbone for AML induction therapy.

Author contributions Jan Braess, Susanne Amler, Christina Sauerland, and Wolfgang Hiddemann designed research, collected data, analyzed and interpreted data, performed statistical analysis, and read and wrote the manuscript. Karl Anton Kreuzer, Karsten Spiekermann, Hans Walter Lindemann, Eva Elisabeth Lengfelder, Ullrich Graeven, Peter Staib, Wolf-Dieter Ludwig, Harald Biersack, Yon-Dschun Ko, Michael Uppenkamp, Maike de Wit, Stefan Korsten, Rudolf Peceny, Tobias Gaska, Xaver Schiel, Dirk Behringer, Michael Kiehl, Bettina Zinngrebe, Gerald Meckenstock, Eva Roemer, Dirk Medgenberg, Ernst Spaeth-Schwalbe, Gero Massenkeil, Heidrun Hindahl, Rainer Schwerdtfeger, Guido Trenn, Stefan Bohlander, Stephanie Schneider, Annika Maria Dufour, Christian Buske, Michael Fiegl, Marion Subklewe, Birgit Braess, Anja Baumgartner, Bernhard Wörmann, and Dietrich Wilhelm Beelen collected data, analyzed and interpreted data, and read and wrote the manuscript. Raphael Koch designed research, analyzed and interpreted data, performed statistical analysis, and read and wrote the manuscript. Martin Lablans implemented randomization and read and wrote the manuscript. Andreas Faldum designed research and read and wrote the manuscript. Dennis Görlich analyzed and interpreted data, performed statistical analysis, and read and wrote the manuscript. Michael Unterhalt designed research, analyzed and interpreted data, performed statistical analysis, and read and wrote the manuscript.

\section{Compliance with ethical standards}

Conflict of interest Jan Braess has received research funding from Amgen and Janssen. Eva Elisabeth Lengfelder has received travel \& accommodation expenses from Teva and Novartis. Ullrich Graeven has received honoraria from Amgen, Roche, Bayer, Sanofi, AbbVie, Servier, and Boehringer; has a consulting or advisory role in Baxalta, Servier, Roche, and Novartis; and has received travel \& accommodation expenses from Merck and Sanofi. Dirk Behringer has a consulting or advisory role in Roche and BMS. Gero Massenkeil has received honoraria from Sanofi; has a consulting or advisory role in Sanofi; and is in speakers' bureau in Sanofi. Stefan Bohlander has received honoraria from Roche. Wolfgang Hiddemann has received honoraria from Roche, Gilead, and Janssen; is in speakers' bureau in Roche, Gilead, and Janssen; has received research funding from Amgen, Roche, Gilead, and Janssen; has received travel \& accommodation expenses from Amgen, Roche, Gilead, and Janssen. All other authors declare that they have no conflict of interest.

Open Access This article is licensed under a Creative Commons Attribution 4.0 International License, which permits use, sharing, adaptation, distribution and reproduction in any medium or format, as long as you give appropriate credit to the original author(s) and the source, provide a link to the Creative Commons license, and indicate if changes were made. The images or other third party material in this article are included in the article's Creative Commons license, unless indicated otherwise in a credit line to the material. If material is not included in the article's Creative Commons license and your intended use is not permitted by statutory regulation or exceeds the permitted use, you will need to obtain permission directly from the copyright holder. To view a copy of this license, visit http://creativecommons. org/licenses/by/4.0/.

\section{References}

1. Dohner H, Estey E, Grimwade D, Amadori S, Appelbaum FR, Buchner T, et al. Diagnosis and management of AML in adults: 2017 ELN recommendations from an international expert panel. Blood. 2017;129:424-47.

2. Stone RM, Mandrekar SJ, Sanford BL, Laumann K, Geyer S, Bloomfield CD, et al. Midostaurin plus chemotherapy for acute myeloid leukemia with a FLT3 mutation. $N$ Engl J Med. 2017;377:454-64.

3. Metzeler KH, Herold T, Rothenberg-Thurley M, Amler S, Sauerland MC, Gorlich D, et al. Spectrum and prognostic relevance of driver gene mutations in acute myeloid leukemia. Blood. 2016;128:686-98.

4. Papaemmanuil E, Gerstung M, Bullinger L, Gaidzik VI, Paschka P, Roberts ND, et al. Genomic classification and prognosis in acute myeloid leukemia. N Engl J Med. 2016;374: 2209-21.

5. Buchner T, Hiddemann W, Wormann B, Loffler H, Gassmann W, Haferlach T, et al. Double induction strategy for acute myeloid leukemia: the effect of high-dose cytarabine with mitoxantrone instead of standard-dose cytarabine with daunorubicin and 6thioguanine: a randomized trial by the German AML Cooperative Group. Blood. 1999;93:4116-24.

6. Buchner T, Krug UO, Peter Gale R, Heinecke A, Sauerland MC, Haferlach $\mathrm{C}$, et al. Age, not therapy intensity, determines outcomes of adults with acute myeloid leukemia. Leukemia. 2016;30:1781-4. 
7. Castaigne S, Chevret S, Archimbaud E, Fenaux P, Bordessoule D, Tilly $\mathrm{H}$, et al. Randomized comparison of double induction and timed-sequential induction to a " $3+7$ " induction in adults with AML: long-term analysis of the Acute Leukemia French Association (ALFA) 9000 study. Blood. 2004;104:2467-74.

8. Schaich M, Parmentier S, Kramer M, Illmer T, Stolzel F, Rollig C, et al. High-dose cytarabine consolidation with or without additional amsacrine and mitoxantrone in acute myeloid leukemia: results of the prospective randomized AML2003 trial. J Clin Oncol. 2013;31:2094-102.

9. Kern W, Schoch C, Haferlach T, Braess J, Unterhalt M, Wormann B. et al. Multivariate analysis of prognostic factors in patients with refractory and relapsed acute myeloid leukemia undergoing sequential high-dose cytosine arabinoside and mitoxantrone (SHAM) salvage therapy: relevance of cytogenetic abnormalities. Leukemia. 2000;14:226-31.

10. Braess J, Spiekermann K, Staib P, Gruneisen A, Wormann B, Ludwig WD, et al. Dose-dense induction with sequential highdose cytarabine and mitoxantone (S-HAM) and pegfilgrastim results in a high efficacy and a short duration of critical neutropenia in de novo acute myeloid leukemia: a pilot study of the AMLCG. Blood. 2009;113:3903-10.

11. Krug U, Berdel WE, Gale RP, Haferlach C, Schnittger S, MullerTidow $\mathrm{C}$, et al. Increasing intensity of therapies assigned at diagnosis does not improve survival of adults with acute myeloid leukemia. Leukemia. 2016;30:1230-6.

12. Buchner T, Berdel WE, Schoch C, Haferlach T, Serve HL, Kienast $\mathrm{J}$, et al. Double induction containing either two courses or one course of high-dose cytarabine plus mitoxantrone and postremission therapy by either autologous stem-cell transplantation or by prolonged maintenance for acute myeloid leukemia. J Clin Oncol. 2006;24:2480-9.
13. Cheson BD, Bennett JM, Kopecky KJ, Buchner T, Willman CL, Estey EH, et al. Revised recommendations of the International Working Group for Diagnosis, Standardization of Response Criteria, Treatment Outcomes, and Reporting Standards for Therapeutic Trials in Acute Myeloid Leukemia. J Clin Oncol. 2003;21:4642-9.

14. Whitehead J. The design and analysis of sequential clinical trials. 3rd ed. John Wiley \& Sons; 1997.

15. Kern W, Aul C, Maschmeyer G, Kuse R, Kerkhoff A, GroteMetke A, et al. Granulocyte colony-stimulating factor shortens duration of critical neutropenia and prolongs disease-free survival after sequential high-dose cytosine arabinoside and mitoxantrone (S-HAM) salvage therapy for refractory and relapsed acute myeloid leukemia. German AML Cooperative Group. Ann Hematol. 1998;77:115-22.

16. Jaramillo S, Benner A, Krauter J, Martin H, Kindler T, Bentz M, et al. Condensed versus standard schedule of high-dose cytarabine consolidation therapy with pegfilgrastim growth factor support in acute myeloid leukemia. Blood Cancer J. 2017;7:e564.

17. Woods WG, Kobrinsky N, Buckley JD, Lee JW, Sanders J, Neudorf $S$, et al. Timed-sequential induction therapy improves postremission outcome in acute myeloid leukemia: a report from the Children's Cancer Group. Blood. 1996;87:4979-89.

18. Woods WG, Neudorf S, Gold S, Sanders J, Buckley JD, Barnard $\mathrm{DR}$, et al. A comparison of allogeneic bone marrow transplantation, autologous bone marrow transplantation, and aggressive chemotherapy in children with acute myeloid leukemia in remission. Blood. 2001;97:56-62.

19. Rollig C, Serve H, Huttmann A, Noppeney R, Muller-Tidow C, Krug U, et al. Addition of sorafenib versus placebo to standard therapy in patients aged 60 years or younger with newly diagnosed acute myeloid leukaemia (SORAML): a multicentre, phase 2, randomised controlled trial. Lancet Oncol. 2015;16:1691-9.

\section{Affiliations}

Jan Braess ${ }^{1,2} \cdot$ Susanne Amler ${ }^{3,4} \cdot K^{\prime}$ arl-Anton Kreuzer ${ }^{5} \cdot K^{\prime}$ arsten Spiekermann ${ }^{2} \cdot$ Hans Walter Lindemann ${ }^{6}$. Eva Lengfelder ${ }^{7}$. Ullrich Graeven ${ }^{8} \cdot$ Peter Staib $^{9} \cdot$ Wolf-Dieter Ludwig $^{10} \cdot$ Harald Biersack $^{11} \cdot$ Yon-Dschun Ko ${ }^{12}$. Michael J. Uppenkamp ${ }^{13} \cdot$ Maike De Wit $^{14} \cdot$ Stefan Korsten $^{15} \cdot$ Rudolf Peceny $^{16} \cdot$ Tobias Gaska $^{17}$ - Xaver Schiel ${ }^{18}$. Dirk M. Behringer ${ }^{19}$. Michael G. Kiehl ${ }^{20}$. Bettina Zinngrebe ${ }^{21}$. Gerald Meckenstock ${ }^{22}$ - Eva Roemer ${ }^{23}$. Dirk Medgenberg $^{24} \cdot$ Ernst Spaeth-Schwalbe $^{25} \cdot$ Gero Massenkeil $^{26} \cdot$ Heidrun Hindahl $^{27} \cdot$ Rainer Schwerdtfeger $^{28}$. Guido Trenn ${ }^{29}$ - Cristina Sauerland ${ }^{3} \cdot$ Raphael Koch $^{3} \cdot$ Martin Lablans $^{3,30} \cdot$ Andreas Faldum $^{3} \cdot$ Dennis Görlich $^{3}$. Stefan K. Bohlander ${ }^{2,31} \cdot$ Stephanie Schneider ${ }^{2} \cdot$ Annika Dufour $^{2} \cdot$ Christian Buske $^{2,32} \cdot$ Michael Fiegl $^{2}$ • Marion Subklewe ${ }^{2} \cdot$ Birgit Braess $^{1,2} \cdot$ Michael Unterhalt $^{2} \cdot$ Anja Baumgartner $^{2} \cdot$ Bernhard Wörmann $^{33}$. Dietrich Beelen ${ }^{34}$. Wolfgang Hiddemann ${ }^{2}$ for the AML-CG

1 Department of Oncology and Hematology, Hospital Barmherzige Brüder, Regensburg, Germany

2 Department of Medicine III, University Hospital LMU Campus Grosshadern, Munich, Germany

3 Insitute for Biostatistics and Clinical Research, University Hospital, Münster, Germany

4 Friedrich Löffler Institute, Federal Research Centre, GreifswaldInsel Riems, Germany

5 Department of Internal Medicine I, University Hospital, Cologne, Germany

6 Department of Hematology and Oncology, Catholic Hospital, Hagen, Germany
Department of Medicine III, University Hospital, Mannheim, Germany

8 Department of Medicine I, Hospital Maria Hilf, Mönchengladbach, Germany

9 Department of Hematology and Medical Oncology, St. Antonius Hospital, Eschweiler, Germany

10 Department of Hematology and Oncology and Tumor Immunology, Helios Hospital, Berlin-Buch, Germany

11 Department of Medicine I, University Hospital, Lübeck, Germany

12 Department of Medicine I, Johanniter Hospital, Bonn, Germany

13 Department of Medicine A, Klinikum Ludwigshafen, Ludwigshafen, Germany 
14 Department of Hematology, Oncology and Palliative Care, Vivantes Klinikum Neukölln, Berlin, Germany

15 Department of Medicine, Vinzenz Pallotti Hospital, BergischGladbach, Germany

16 Department of Hematology and Oncology, Klinikum Osnabrïck, Osnabrück, Germany

17 Department of Hematology and Oncology, St. Josef Hospital, Paderborn, Germany

18 Department of Hematology and Oncology, Klinikum Harlaching, Munich, Germany

19 Department of Hematology, Oncology and Palliative Care, Augusta Hospital, Bochum, Germany

20 Department of Medicine I, Klinikum Frankfurt/Oder, Frankfurt/ Oder, Germany

21 Department of Hematology, Oncology and Palliative Care, Evangelisches Krankenhaus, Bielefeld, Germany

22 Department of Medical Oncology, Radiooncology, Hematology and Palliative Care, St. Josef Hospital, Gelsenkirchen, Germany

23 Department of Hematology and Oncology, Klinikum IdarOberstein, Idar-Oberstein, Germany

24 Department of Medicine III, Klinikum Leverkusen,
Leverkusen, Germany

25 Department of Medicine, Vivantes Klinikum Spandau, Berlin, Germany

26 Department of Medicine II, Klinikum Gütersloh, Gütersloh, Germany

27 Department of Medicine I, St. Johannes Hospital, Dortmund, Germany

28 Department for Bone Marrow and Blood Stem Cell Transplantation, DKD Deutsche Klinik für Diagnostik, Wiesbaden, Germany

29 Department of Medicine I, Knappschaftskrankenhaus, Bottrop, Germany

30 Division of Medical Informatics in Translational Oncology, DKFZ German Cancer Research Center, Heidelberg, Germany

31 Department of Molecular Medicine and Pathology, University of Auckland, Auckland, New Zealand

32 Institute of Experimental Cancer Research, University Hospital, Ulm, Germany

33 German Society of Hematology and Oncology DGHO, Berlin, Germany

34 Department of Bone Marrow Transplantation, University Hospital, Essen, Germany 